\title{
Measures of co-expression for improved function prediction of long non-coding RNAs
}

Rezvan Ehsani ${ }^{1,2^{*}}$ and Finn Drabløs ${ }^{3^{*}}$ (D)

\begin{abstract}
Background: Almost 16,000 human long non-coding RNA (IncRNA) genes have been identified in the GENCODE project. However, the function of most of them remains to be discovered. The function of IncRNAs and other novel genes can be predicted by identifying significantly enriched annotation terms in already annotated genes that are co-expressed with the IncRNAs. However, such approaches are sensitive to the methods that are used to estimate the level of co-expression.

Results: We have tested and compared two well-known statistical metrics (Pearson and Spearman) and two geometrical metrics (Sobolev and Fisher) for identification of the co-expressed genes, using experimental expression data across 19 normal human tissues. We have also used a benchmarking approach based on semantic similarity to evaluate how well these methods are able to predict annotation terms, using a wellannotated set of protein-coding genes.

Conclusion: This work shows that geometrical metrics, in particular in combination with the statistical metrics, will predict annotation terms more efficiently than traditional approaches. Tests on selected IncRNAs confirm that it is possible to predict the function of these genes given a reliable set of expression data. The software used for this investigation is freely available.
\end{abstract}

Keywords: Function prediction, Gene annotation, Co-expression, Fisher information metric, Sobolev metric, semantic similarity

\section{Background}

Long non-coding RNAs (lncRNAs), defined as non-protein-coding transcripts longer than 200 nucleotides, are one of the most common RNA species, but they are in most cases poorly understood with respect to function [1]. It has been shown that IncRNAs play important roles in a wide range of biological process [2, 3] and diseases [4-6]. Possible functions of lncRNAs can be characterized experimentally using gain- and loss-of-function approaches $[7,8]$, but this is not a straightforward method, for example because lncRNAs can be expressed in multiple isoforms. Therefore, to apply computational methods and algorithms can be a

\footnotetext{
*Correspondence: rezvanehsani74@gmail.com; finn.drablos@ntnu.no ${ }^{1}$ Department of Mathematics, University of Zabol, Zabol, Iran

${ }^{3}$ Department of Clinical and Molecular Medicine, NTNU - Norwegian University of Science and Technology, NO-7491 Trondheim, Norway Full list of author information is available at the end of the article
}

good and accessible supplement to experimental methods for suggesting possible functions of lncRNAs and other un-annotated genes.

Currently, such computational approaches are still at an early stage of development, although its importance has been recognized [9-11]. There are considerable challenges in finding precise and reliable computational approaches due to a lack of suitable data. There is also a lack of databases with relevant features that are suitable for example for machine learning, there is a lack of lncRNAs with known function for training, and for most lncRNAs we are not aware of any common structural features that are important for function. For example, many lncRNA gene sequences are not conserved and do not contain clear motifs [12], which makes it difficult to find and predict the function of lncRNAs by relying on their sequences. A lack of any rich set of molecular

(c) The Author(s). 2018 Open Access This article is distributed under the terms of the Creative Commons Attribution 4.0 International License (http://creativecommons.org/licenses/by/4.0/), which permits unrestricted use, distribution, and reproduction in any medium, provided you give appropriate credit to the original author(s) and the source, provide a link to the Creative Commons license, and indicate if changes were made. The Creative Commons Public Domain Dedication waiver (http://creativecommons.org/publicdomain/zero/1.0/) applies to the data made available in this article, unless otherwise stated. 
interaction data for most lncRNAs is also a limitation with respect to computational annotation [13, 14].

One possible and frequently used approach is based on "guilt by association", i.e., to identify well-annotated genes that seem to be involved in some of the same processes as a given un-annotated gene. This association is often based on co-expression, indicating potential co-regulation of a set of genes $[15,16]$. It is then possible to predict function of the un-annotated gene by using information from the well-annotated co-expressed genes, assuming that co-expressed genes may be involved in similar or at least related processes. This approach is illustrated in Fig. 1, and it has been tested in several implementations, with some success. Alam et al. and Gong et al. list several methods and databases for annotation of ncRNAs [17, 18], and most of these use co-expression at some stage, mainly with standard statistical metrics like Pearson or Spearman. For example, Guttman et al. determined several sets of mouse lncRNAs to be related to sets of mRNA for protein-coding genes by Pearson correlation [19], and co-expressed lncRNA-mRNA networks for mouse have also been used by Guo et al. (with "Inc-GFP") and Liao et al. (using a coding-non-coding $(\mathrm{CNC})$ network computed with Pearson correlation) to assigned function to 340 and 1625 mouse lncRNAs, respectively [20,21]. Jiang et al. used Pearson correlation to identify co-expressed genes for human IncRNAs (with "LncRNA2Function"), and they could annotate given lncRNAs with significantly enriched gene ontology (GO) terms among the set of co-expressed protein-coding genes, according to the hypergeometric test [22]. Park et al. built a database of lncRNAs ("IncRNAtor"), where they included co-expression by Spearman correlation [23], whereas Zhao et al. developed a web-based application ("CoLncRNA") for exploring combinatorial effects of lncRNAs, using linear regression and Spearman correlation to map co-expression between lncRNAs and protein-coding genes [24]. Perron et al. combined co-expression by Pearson correlation with evolutionary conservation in "FuncPred" [25], whereas Zhou et al. used a combination of ChIP-seq, CLIP-seq and RNA-seq (in "IncFunNet") to predict IncRNA function, using Pearson correlation to estimate co-expression of gene pairs [26]. Zhou et al. also released a toolkit ("IncFunTK") for calculation of a Functional Information Score (FIS) [27], based on their previous work with lncFunNet. Recently Zhang et al. used a hierarchy of neural networks to predict GO terms for lncRNA genes, implemented as "NeuraNetL2GO", with Pearson correlation as the measure of co-expression [28].

Thus, most proposed methods rely on identification of co-expressed genes from experimental expression data. Therefore, estimation of co-expression is a crucial step, and relevant alternative metrics should be evaluated. Here we use several metrics for estimating co-expression, in particular statistical (Pearson, Spearman) and geometrical ones (Sobolev, Fisher), and a combination of those. This has been implemented as LNCRNA2GOA, which is available to users. The aim is to provide improved identification of true co-expression. We use an enrichment analysis to identify enriched GO-terms in the co-expressed gene sets, and use this to predict GO terms for the un-annotated gene. We have benchmarked the methods for co-expression on a subset of well-annotated proteincoding genes, using semantic similarity to compare real

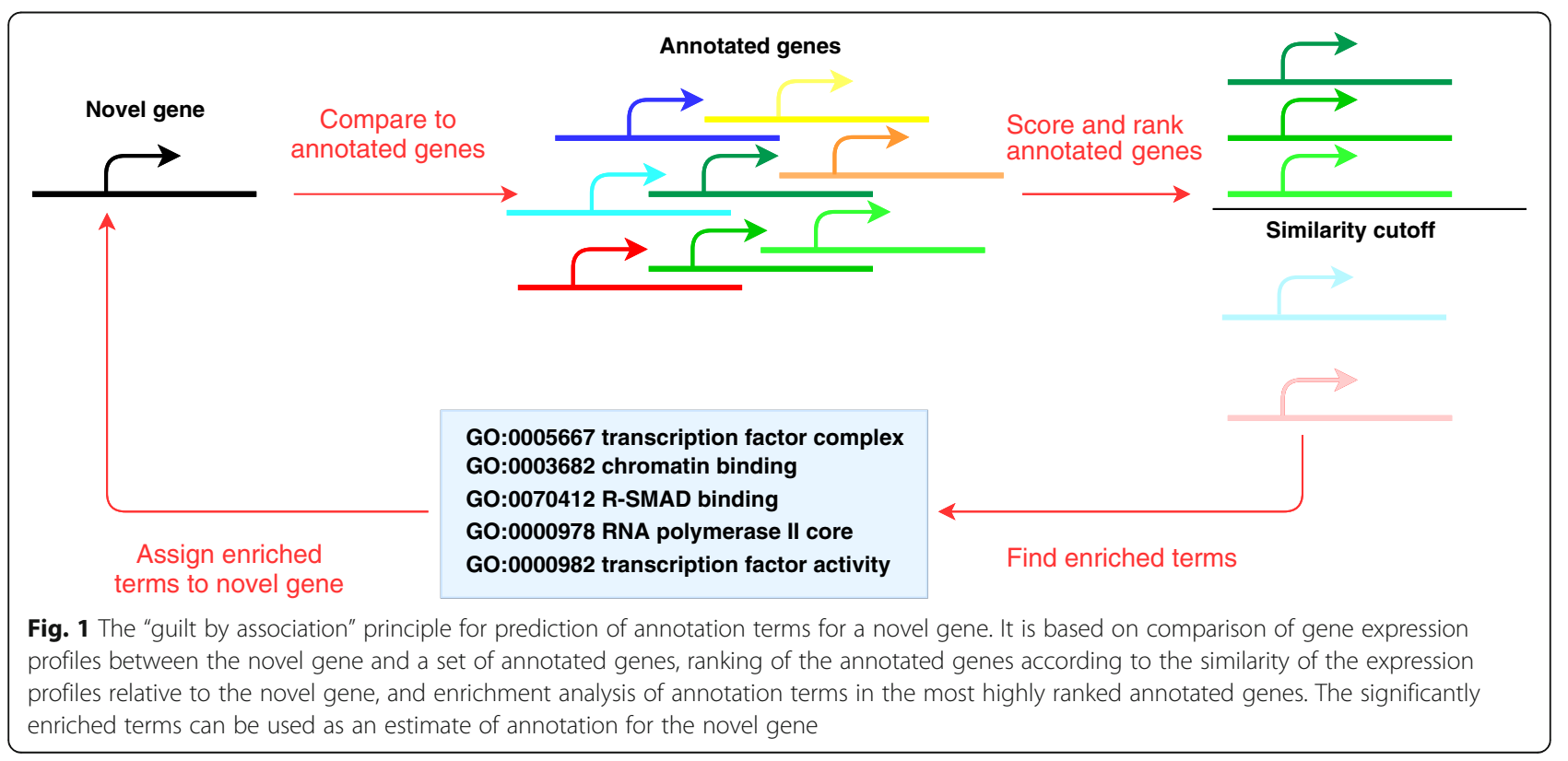


and predicted GO terms, and also tested the performance on a small number of well-known human lncRNAs.

\section{Methods}

\section{Data sources}

For expression data we have used data from Jiang et al. [22]. They used the information from GENCODE V15 [29] for genomic coordinates and RNA-Seq data of 19 human normal tissues from the Human Body Map 2 project (ArrayExpress accession GSE0554), and read and computed expression values using tophat [30] and cufflinks [31] for all human IncRNA and protein-coding genes. Details are given in the paper by Jiang et al. [22]. GO annotations were downloaded from the Gene Ontology Project [32], and R [33] version 3.3.3, Plyr [34] version 1.8.5, and GOSim [35] version 1.12.0 were used for the implementation.

\section{Main workflow}

The schematic workflow is shown in Additional file 1: Figure S1, where key aspects of this study (i.e., metrics for comparison of expression profiles, and similarity measures for comparing predicted and known annotations) are highlighted. A pseudocode representation of the LNCRNA2GOA algorithm is shown in Table 1. The goal is function prediction for an lncRNA or another poorly annotated gene (denoted as $g$ ). Let be a set of statistical and geometrical methods (see below) and Target $_{g}^{m}$ be all protein-coding (or well-annotated) genes that are co-expressed with $g$ as determined by method $m$. Now the gene $g$ will be functionally annotated with significantly enriched annotation terms (here GO) among the set of co-expressed well-annotated proteincoding genes. We use the hypergeometric test to compute the $p$-value of each term $T$ :

$$
p=\frac{\sum_{i=t}^{\min (n, M)}\left(\begin{array}{c}
M \\
i
\end{array}\right)\left(\begin{array}{c}
N-M \\
n-i
\end{array}\right)}{\left(\begin{array}{c}
N \\
n
\end{array}\right)}
$$

Herein, $N$ is the number of all protein-coding genes, $M$ is the number of protein-coding genes that are annotated in the functional term $T, n$ is the size of Target $_{g}^{m}$ and $t$ is the number of genes in Target ${ }_{g}^{m}$ that are annotated in the functional term $T$. Because the statistical analysis is not appropriate for problems of small size, we exclude GO terms with less than five annotated protein-coding genes from the enrichment analysis, as recommended by Jiang et al. [22]. We also use false discovery rate (FDR) for correction for multiple hypothesis tests. The significance cut-off of corrected $p$-value is set as 0.05 .

\section{Methods to identify Target $\mathrm{g}_{\mathrm{g}}^{\mathrm{m}}$}

To identify co-expressed genes for a typical gene $g$, we used the geometrical metrics Sobolev and Fisher information, in addition to the statistical metrics Pearson and Spearman.

\section{Sobolev metric}

In this section, we use definitions and notations as in [36]. We start with the usual p-inner product. Let $f, g$ be real-valued functions (in this case $f$ and $g$ values are the expression vectors of two genes $f$ and $g$ ):

$$
f, g_{p}=\left(\sum_{k=1}^{n}\left|f_{k} \cdot g_{k}\right|^{p}\right)^{\frac{1}{p}}
$$

By this notation, Sobolev inner product, norm and meter of degree $k$ respectively can be defined by:

$$
\begin{aligned}
& \langle f, g\rangle_{p, \alpha}^{S}=\langle f, g\rangle_{p}+\alpha\left\langle D^{k} f, D^{k} g\right\rangle_{p} \\
& \|f\|_{p, k, \alpha}^{S}=\sqrt{\langle f, f\rangle_{p, \alpha}^{S}} \\
& d_{p, k, \alpha}^{S}(f, g)=\|f-g\|_{p, k, \alpha}^{S}
\end{aligned}
$$

where $D^{k}$ is the $k$ th differential operator. For the special case $p=2$ and $\alpha=1$ an interesting connection to the Fourier-transform of analysis can be made; let $\hat{f}$ be the Fourier-transform $f$

$$
\hat{f}\left(\omega_{k}\right)=\sum_{j=1}^{N-1} g_{j} \exp \left(-i \frac{2 \pi k j}{N}\right)
$$

where $\omega_{k}=\frac{2 \pi k}{N}$ and $i=\sqrt{-1}$. Finally, the norm can be written as

$$
\|f\|_{2, k, 1}^{S}=\sqrt{\sum_{j=1}^{N-1}\left(1+\omega_{j}\right)^{k}\left|\hat{f}\left(\omega_{j}\right)\right|^{2}}
$$

In this work we used metric (5) with norm (7) and $k=1$.

\section{Fisher information metric}

In this section we use definitions and notations as in [37]. To define Fisher information metric we first introduce the $n$-simplex $P_{n}$ defined by

$$
P_{n}=\left\{x \in R^{n+1}: \forall i, x_{n} \geq 0, \sum_{i=1}^{n+1} x_{i}=1\right\}
$$

The coordinates $\left\{x_{i}\right\}$ describe the probability of observing different outcomes in a single experiment (or expression value of a gene in $i$ th cell type). The Fisher information metric on $P_{n}$ can be defined by 
Table 1 Pseudocode of the LNCRNA2GOA algorithm

Algorithm 1 - LNCRNA2GOA

$\overline{\text { Required: }}$ EX: Data frame of all expression values of protein-coding (EXPC) and lncRNA genes

EG: Data frame of Ensembl ID and GO ID for protein-coding genes
Input: $\quad$ : Ensembl ID; Onto: Ontology type; m: Method
Output: Data frame of all significant terms ordered by FDR

Function Enrich(m)

Score_ $\mathrm{m} \leftarrow$ all possible co-expression values between $\mathrm{g}$ and EXPC genes are computed, using $\mathrm{m}$

$\mathrm{g}$ is filtered out from Score_ $\mathrm{m}$ (to enable leave-one-out)

TopGenes $\leftarrow$ genes are ordered by Score_m and top 250 genes are selected

GO IDs are added to TopGenes, using EG

Enrich_m $\leftarrow$ enrichment of GO is computed for TopGenes by hypergeometric score

Return Enrich_m

End Enrich

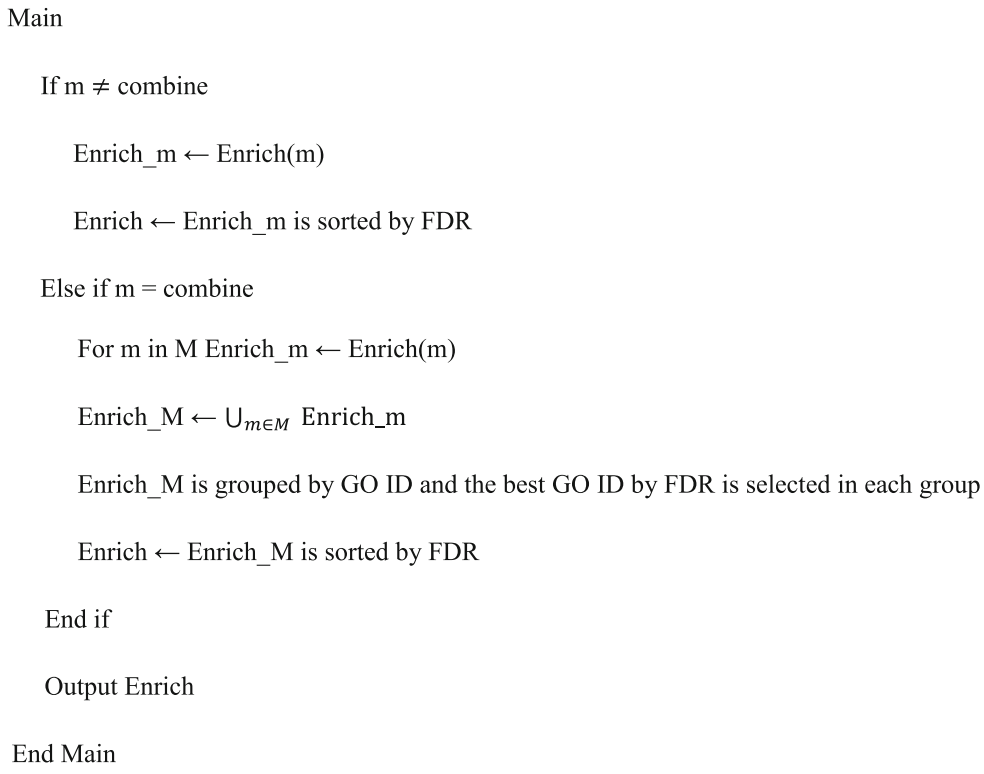

$$
J_{i j}=\sum_{k=1}^{n+1} \frac{1}{x_{k}} \frac{\partial x_{k}}{\partial x_{i}} \frac{\partial x_{k}}{\partial x_{j}}
$$

We now define a well-known representation of the Fisher information as a pull-back metric from the positive $n$-sphere $S_{n}^{+}$;

$$
S_{n}^{+}=\left\{x \in R^{n} ; \forall i, x_{n} \geq 0, \sum_{i=1}^{n+1} x^{2}=1\right\}
$$

The transformation $T: P_{n} \rightarrow S_{n}^{+}$defined by 


$$
T(x)=\left(\sqrt{x_{1}}, \ldots, \sqrt{x_{n+1}}\right)
$$

pulls back the Euclidean metric on the surface of the sphere to the Fisher information on the multinomial simplex. Actually, the geodesic distance for $x, y \in P_{n}$ under the Fisher information metric may be defined by measuring the length of the great circle on $S_{n}^{+}$between $T(x)$ and $T(y)$

$$
d(x, y)=\operatorname{acos}\left(\sum_{i=1}^{n+1} \sqrt{x_{i} y_{i}}\right)
$$

\section{Size of Target ${ }_{g}^{m}$}

In the previous section we introduced four methods to measure similarity between genes based on their expression values, which can be used to rank genes. Now the challenge is to determine a threshold for identifying the most informative set of correlated genes as Target $_{g}^{m}$. Some studies, for example [22] used a threshold of 0.9 on the Pearson metric, but this does not identify an optimal cutoff; sometimes it returns thousands of co-regulated genes, and sometimes nothing. It is also difficult to set a threshold in a similar way when using a geometrical metric. Therefore, we tried to select a threshold based on a possible number of Target $_{g}^{m}$ elements. We selected a range of different sizes for subsets of the most similar genes from the Target $t_{g}^{m}$ set, including $\{50 * x \mid x=1,2, \ldots, 10\}$, and analysed how well the algorithm could predict some well-known lncRNAs. This analysis showed that a selection of the top 250 co-expressed protein-coding genes seemed to have optimal performance for prediction of GO terms for some well-known lncRNAs.

\section{Combination of methods}

Let $\operatorname{SigGOs}_{g}^{m, r}=\left[\operatorname{SigGOs}_{g}^{m}\right]^{r}$ be the $r$ most significant terms assigned to gene $g$ by method $m$. Since the optimal method $m$ is individually different we can assign $\operatorname{SigGOs} s_{g}^{r, s}=\left[\cup_{m \in \mathrm{M}} \operatorname{SigGOS}_{g}^{m, r}\right]^{s}$ to the gene, and this will increase the accuracy of predictions (see Results and discussion). If there are identical terms for some methods, we just consider the term with the minimum corrected $p$-value. Actually, in the combination method the algorithm first collects the $r$ most significant terms for all $m \in \mathrm{M}$, then selects the $s$ most significant terms from this collection. When $r=$ all and $s=$ all then the algorithm returns all significant terms.

\section{Evaluation on protein-coding genes}

Before evaluating the performance of our method on some known lncRNA genes, we benchmarked it on a set of well-annotated genes. Since protein-coding genes in general are much more well-annotated than lncRNA genes, this set was based on protein-coding genes. We selected protein-coding genes annotated by 5 or 6 molecular function (MF) terms and 9 or 10 biological process (BP) terms in the GO database. This represents the average number of GO-terms in each set, as the genes were on average annotated by $4.67 \mathrm{MF}$ and $9.25 \mathrm{BP}$ terms. Therefore the benchmark set has a typical (average) level of annotation. It consists of 352 protein-coding genes (denoted as Test $_{352}$ ), and is available as part of the LNCRNA2GOA distribution [38]. We used a "leave-one-out" approach for the actual benchmarking. That is, for each gene in Test 352 we treated the gene as unannotated and predicted GO terms for the gene by each of five different methods (Pearson, Spearman, Sobolev, Fisher, and Combine, which is a combination of all four methods). We then used the TopoICSim [39] and GOSemSim [40] approaches to estimate similarity between real and predicted GO annotations for each gene in Test ${ }_{352}$. TopoICSim and GOSemSim are two algorithms for measuring semantic similarity between pairs of genes. To have a more realistic semantic measure for benchmarking, we restricted the size of the output set of enriched terms by selecting $\operatorname{SigGOs}(m, 2 \times L)$ where $L$ is the number of GO terms for gene $g$ in Test 352 based on the gene ontology database. For the Combine case, where we find an optimal combination of predictions from each of the four different methods, we evaluated the performance first for SigGOs $\mathrm{M}_{g}^{\mathrm{M}, 2 \times L, 2 \times L} \quad$ (Combine_2L) and then For SigGOs $s_{g}^{\mathrm{M}, 2 \times L, 4 \times L}$ (Combine_4L).

\section{Results}

Evaluation on Test ${ }_{352}$

Before applying LNCRNA2GOA to human lncRNAs, we first benchmarked the performance on the wellannotated set of protein-coding genes, Test $_{352}$. We have applied all metrics in for each gene in Test 352 and evaluated semantic similarity between actual and predicted annotation as measured by the GOSemSim and TopoICSim methods. We also included results for LncRNA2Function [22] and Co-LncRNA [24]. The results for Co-LncRNA were based on the published computational approach, but using the LncRNA2Function database, to keep the results comparable. The average semantic similarities for each method and metric are shown in Fig. 2. In all cases the TopoICSim measure shows better performance than GOSemSim. It has previously been shown that TopoICSim seems to be a more correct measure for semantic similarity compared to other approaches [39], therefore this most likely reflects a real similarity in predicted annotation, and not a systematic bias in 


\section{a GoSemSim}

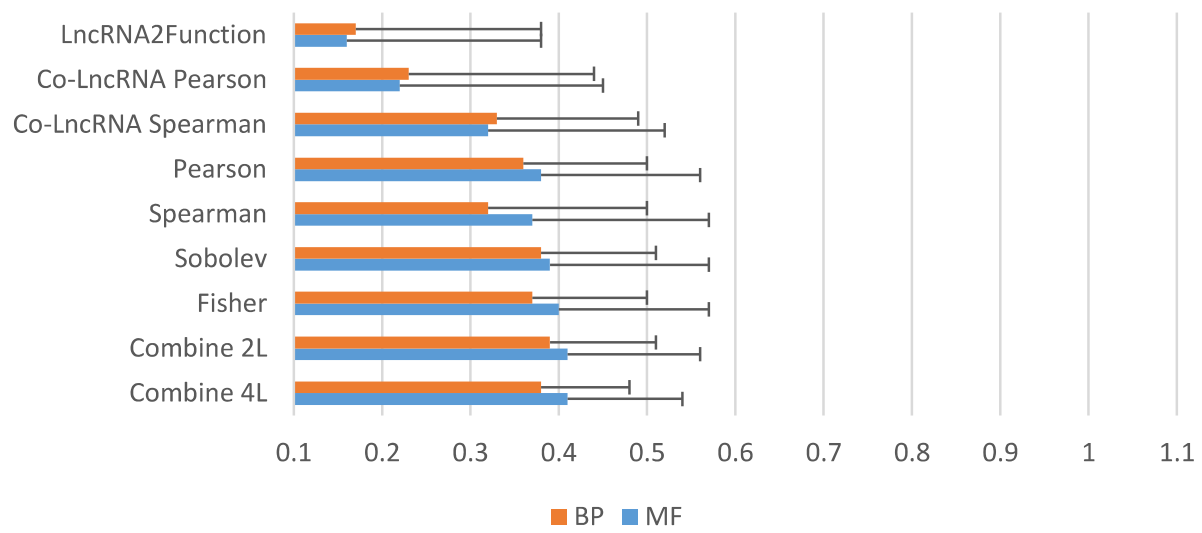

\section{b TopoICSim}

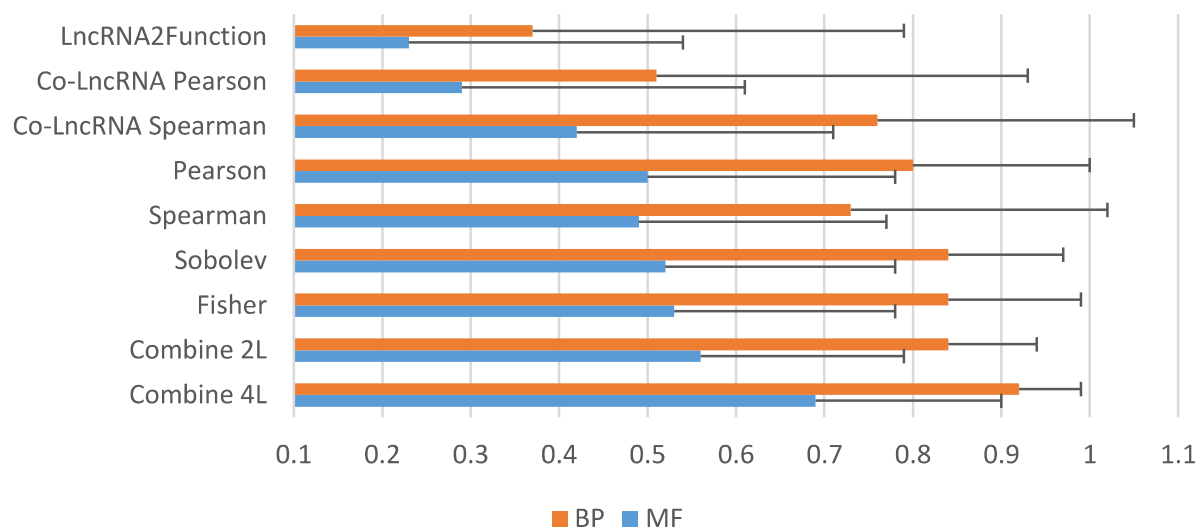

Fig. 2 Evaluation of the different similarity metrics for 352 well-annotated protein-coding genes (Test 352 ). For each gene in Test 352 all methods were applied for prediction of function, and similarity between real and predicted terms were measured with TopolCSim and GOSemSim. The table shows average similarity scores over the test set, with standard deviation. LncRNA2Function: Co-expressed protein-coding genes was obtained for each gene in Test 352 using [22] with Pearson correlation coefficient > 0.9. Co-LncRNA_Pearson or _Spearman: Co-expressed protein-coding genes was obtained for each gene in Test ${ }_{352}$ using [24] with Pearson or Spearman correlation coefficient $>0.8$ and LncRNA2Function expression data

measurements. In particular for TopoICSim there is a good correlation between performance in MF and BP, indicating that the improved performance is not random, but due to the choice of better methods. The results also show that the geometrical metrics predict function more efficiently than statistical metrics, and in particular the combined measure shows quite good performance. It can be argued that the standard deviation (SD) of the averages is high, indicating a variation in performance. However, the SD is lower for the Combine measure. The overall performance seems to be better for BP compared to MF. It is possible that this reflects a bias in the dataset, as each gene in Test $_{352}$ has almost twice as many BP terms compared to MF terms. In summary, Fig. 2 shows that the combined approach has good performance with respect to prediction of $\mathrm{GO}$ terms, in particular for terms related to BP.

\section{Evaluation on data from FuncPred}

We also tested the performance of LNCRNA2GOA on a set of 37 manually annotated lncRNAs from FuncPred [25]. Figure 3 shows the number of successful predictions by LNCRNA2GOA, FuncPred and LncRNA2Function [22]. Here a prediction was counted as successful if at least one of the correct GO terms could be predicted. The same approach has previously been used for example in testing of the NeuraNetL2GO method [28]. Again we see that the best performance was achieved with the LNCRNA2GOA approach.

\section{Comparison to functional predictions by IncFunTK}

Finally, we have done a qualitative evaluation of predictions performed with LNCRNA2GOA, compared to predictions by lncFunTK with data from HeLa cells and Human Body Map, provided as Table S2 in Zhou et al. [27]. The IncFunTK predictions are sorted according to 


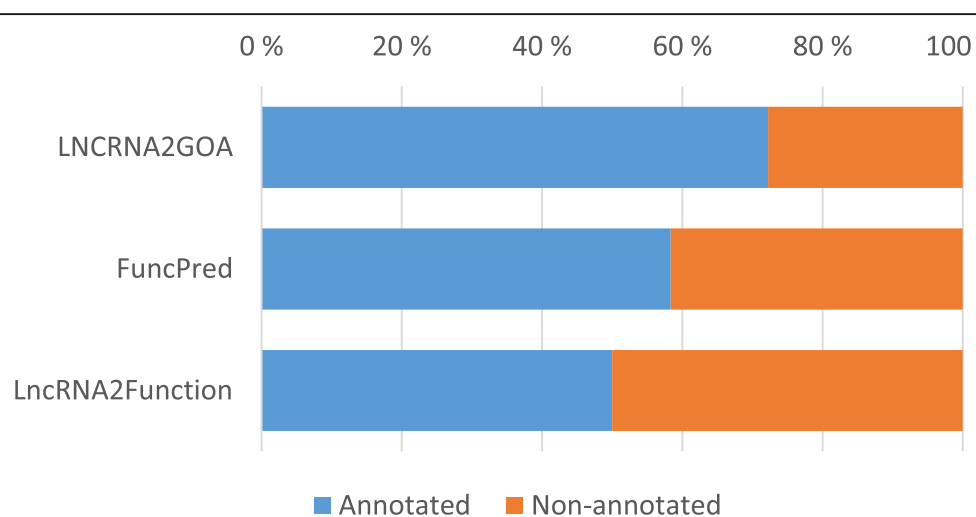

Fig. $3 \mathrm{GO}$ terms for a manually annotated set of 37 IncRNAs from FuncPred has been predicted using LNCRNA2GOA, FuncPred and LncRNA2Function, and the number of successful predictions has been counted

Functional Information Score (FIS), which is a predicted functional importance of a given lncRNA, based on a combination of several data types (ChIP-seq, CLIP-seq, RNA-seq). We have focussed on the 100 most significant cases from lncFunTK according to FIS, and used LNCRNA2GOA to predict GO terms for 50 of those genes where Ensembl IDs could reliably be assigned (see Additional file 2: Table S1). The predicted GO terms were then compared to the GO terms from lncFunTK, using experimental data from literature as a common reference. We were able to retrieve PubMed entries based on gene name for only 20 of the 50 genes, which limits the comparison. However, for more than half of these genes the predictions from LNCRNA2GOA showed at least some similarity to literature data (see Additional file 3: Table S2). For lncFunTK, on the other hand, the available predictions consist of only a single GO term per gene, and most of these terms are identical. For the set of 50 genes, 35 entries are classified as GO:0045944 (positive regulation of transcription from RNA polymerase II promoter) by lncFunTK, and this strong preference for GO:0045944 is the same for the full set of predictions.

It is difficult to do a direct comparison of predictions from these two methods. Both use RNA-seq data from multiple tissue types to estimate co-expression. However, the lncFunTK approach also ranks genes using additional data (in this case, on HeLa cells), which may give some preference for properties that are particularly enriched in this cell type (for example increased transcription). LNCRNA2GOA may to a larger extent display properties that are important across the full range of activities where each lncRNA is involved. This may explain some of the differences in the output between IncFunTK and LNCRNA2GOA. However, it is our general impression that the predictions from LNCRNA2GOA contain more information, compared to lncFunTK, and that several of these predictions seem to be consistent with observations from literature.

\section{Functional annotation of human IncRNAs}

There is a lack of good "gold standard" datasets for human lncRNAs with known function that can be used for benchmarking. However, we have used a small set of five well-known lncRNAs from literature as examples to show the efficiency of LNCRNA2GOA. These lncRNAs have previously been used for documenting the performance of in particular LncRNA2Function [22]. The summary information describing these example lncRNAs, their functions and references are shown in Table 2.

To examine whether LNCRNA2GOA is able to functionally annotate the IncRNAs that are listed in Table 2, we applied the algorithm with defaults (i.e., ontology set as Biological Process and using the Combine method with ( $\left.\operatorname{SigGOs} s_{g}^{\mathrm{M}, \text { all,all }}\right)$, and prediction results are presented and discussed for each lncRNA separately. The top 10 predictions are in each case are given in Table 3.

\section{HOTAIR}

HOTAIR (Hox transcript antisense RNA) is an lncRNA known to be involved in development, cancer and high risk metastases, at least partly through interaction with PRC2 and regulation of HOX genes [41, 42]. To investigate whether LNCRNA2GOA is able to associate functionally relevant GO terms with HOTAIR, we applied the algorithm with defaults parameters. This identified 124 GO BP terms in total. As expected, most significantly enriched terms are associated with development and morphogenesis, and cell metastasis. The results in Table 3 indicate that LNCRNA2GOA successfully identifies functionally relevant GO terms for HOTAIR.

\section{HCP5}

HCP5 (HLA complex P5) is an lncRNA associated with immune response, and it is associated with for example AIDS [43] and virus-related cancers [44]. When testing whether HCP5 can be annotated by LNCRNA2GOA, we 
Table 2 Summary information describing the five case studies for human IncRNAs

\begin{tabular}{|c|c|c|c|}
\hline Ensembl ID & Gene symbol & $\begin{array}{l}\text { Name } \\
\text { Function }\end{array}$ & Selected references \\
\hline ENSG00000228630 & HOTAIR & $\begin{array}{l}\text { Hox transcript antisense RNA } \\
\text { Development process and morphogenesis, cancer } \\
\text { metastasis and invasiveness }\end{array}$ & {$[41,42]$} \\
\hline ENSG00000206337 & $\mathrm{HCP5}$ & $\begin{array}{l}\text { HLA complex P5 } \\
\text { Immune responses }\end{array}$ & {$[43,44]$} \\
\hline ENSG00000251164 & HULC & $\begin{array}{l}\text { Heptacellular carcinoma up-regulated long non-coding } \\
\text { RNA } \\
\text { Liver cancer and colorectal carcinomas that metastasize } \\
\text { to the livers }\end{array}$ & {$[45,46]$} \\
\hline ENSG00000130600 & H19 & $\begin{array}{l}\text { Imprinted maternally expressed transcript } \\
\text { Infertility and multiple cancers such as breast, cervical, liver } \\
\text { and bladder }\end{array}$ & {$[47,48]$} \\
\hline ENSG00000225937 & PCA3 & $\begin{array}{l}\text { Prostate cancer associated } 3 \\
\text { Prostate cancer }\end{array}$ & {$[49,50]$} \\
\hline
\end{tabular}

found that HCP5 was associated with $333 \mathrm{GO}$ terms for biological processes. As expected, most of them involve the immune system and immune response, and functional terms that are associated with the development of AIDS (see Table 3). A relevant example is regulation of immune response (GO:0050776).

\section{HULC}

HULC (Heptacellular carcinoma up-regulated long non-coding RNA) is known to be upregulated in liver cancer and associated with tumorigenesis [45, 46]. We used LNCRNA2GOA to assess whether HULC can be correctly assigned to have liver-related functions. The enrichment output showed 177 GO terms for biological processes associated with HULC. Most of these terms are involved in functions related to liver and lipids, such as lipid hydroxylation (GO:0002933) and chylomicron remodelling (GO:0034371). Table 3 shows the top 10 GO functional terms enriched in protein-coding genes that are co-expressed with the liver-related lncRNA HULC.

\section{H19}

H19 (Imprinted maternally expressed transcript) is known to be important for fertility and several processes associated with female disease risk, including cancer [47, 48]. LNCRNA2GOA identified 53 GO terms for biological processes as associated with $\mathrm{H} 19$. Several of these terms suggested strongly that $\mathrm{H} 19$ can play an important role in infertility or breast cancer, such as female pregnancy (GO:0007565), female gamete generation (GO:0007292), and adrenal gland development (GO:0030325). There were also other relevant GO terms, as for example, JAK-STAT cascade involved in growth hormone signalling pathway (GO:0007565), cell-cell signalling (GO:0007267), and cell proliferation (GO:0008283). This indicates that H19 can play a role in various cancers and other conditions where JAK-STAT signalling is important. The top 10 GO terms for biological processes are shown in Table 3.

\section{PCA3}

PCA3 (Prostate cancer associated 3) is strongly upregulated in prostate cancer $[49,50]$. LNCRNA2GOA identified 25 terms for GO biological processes as associated with PCA3. There were three terms directly involved in prostate cancer; urinary bladder development (GO:0060157), prostate gland development (GO:0030850), and prostate epithelial cord arborization involved in prostate glandular acinus morphogenesis (GO:0060527). PCA3 is a quite challenging case, but this prediction seems to be an improvement over a previous prediction by LncRNA2Function, where only a single pathway linked to androgen receptor was identified, and in some aspects also predictions by the more recent FARNA tool [17].

\section{Additional tests}

We also tested LNCRNA2GOA on a few cases not included in the original LncRNA2Function test set, and got similar results. The two most significant GO BP terms for MALAT1 (Metastasis associated lung adenocarcinoma transcript 1) were ion transport (GO:0006811) and excretion (GO:0007588), which seems to be consistent with the observation that MALAT1 often is associated with kidney function and with renal cell carcinoma [51]. For LINC00152, also known as CYTOR (Cytoskeleton regulator RNA), the two most significant terms were cell adhesion (GO:0007155) and extracellular matrix organisation (GO:0030198), which is consistent with the observation that this lncRNA influences the properties of breast cancer cells with respect to for example invasion and migration [52]. For LINC-ROR (Long intergenic non-protein coding RNA, regulator of reprogramming) the two most significant terms were chromatin silencing at rDNA 
Table 3 Top 10 biological processes assigned to each of the selected case studies

\begin{tabular}{|c|c|c|c|}
\hline GO ID & $P$-value & FDR & Term \\
\hline \multicolumn{4}{|c|}{ HOTAIR (development and morpogenesis) } \\
\hline GO:0032964 & $0.00 \mathrm{e}+00$ & $0.00 \mathrm{e}+00$ & collagen biosynthetic process \\
\hline GO:0030199 & $1.72 \mathrm{e}-14$ & $9.36 \mathrm{e}-12$ & collagen fibril organization \\
\hline GO:0030198 & 1.39e-09 & $3.98 \mathrm{e}-07$ & extracellular matrix organization \\
\hline GO:0007275 & 2.07e-07 & $6.27 e-05$ & multicellular organism development \\
\hline GO:0035115 & $5.31 \mathrm{e}-07$ & 7.63e-05 & embryonic forelimb morphogenesis \\
\hline GO:0060272 & $8.21 \mathrm{e}-07$ & $1.00 \mathrm{e}-04$ & embryonic skeletal joint morphogenesis \\
\hline GO:0048704 & $7.01 \mathrm{e}-07$ & $1.49 \mathrm{e}-04$ & embryonic skeletal system morphogenesis \\
\hline GO:0001568 & $2.13 e-06$ & $1.88 \mathrm{e}-04$ & blood vessel development \\
\hline GO:0007506 & 7.72e-06 & $1.00 \mathrm{e}-03$ & gonadal mesoderm development \\
\hline GO:0002063 & $1.58 \mathrm{e}-05$ & $1.01 \mathrm{e}-03$ & chondrocyte development \\
\hline \multicolumn{4}{|c|}{ HCP5 (immune- and AIDS-related processes) } \\
\hline GO:0002480 & $0.00 \mathrm{e}+00$ & $0.00 \mathrm{e}+00$ & $\begin{array}{l}\text { antigen processing and presentation of exogenous } \\
\text { peptide antigen via MHC class I, TAP-independent }\end{array}$ \\
\hline GO:0002504 & $0.00 \mathrm{e}+00$ & $0.00 \mathrm{e}+00$ & $\begin{array}{l}\text { antigen processing and presentation of peptide or } \\
\text { polysaccharide antigen via MHC class II }\end{array}$ \\
\hline GO:0002376 & $1.35 \mathrm{e}-55$ & $3.37 e-53$ & immune system process \\
\hline GO:0050776 & $1.10 \mathrm{e}-53$ & $1.22 \mathrm{e}-50$ & regulation of immune response \\
\hline GO:0006955 & $6.98 \mathrm{e}-50$ & $1.39 \mathrm{e}-47$ & immune response \\
\hline GO:0002250 & $6.06 \mathrm{e}-28$ & $1.35 \mathrm{e}-25$ & adaptive immune response \\
\hline GO:0050852 & $3.56 \mathrm{e}-27$ & $5.93 e-25$ & T cell receptor signaling pathway \\
\hline GO:0019882 & 7.67e-27 & $1.09 \mathrm{e}-24$ & antigen processing and presentation \\
\hline GO:0045087 & $6.77 e-23$ & $1.26 \mathrm{e}-20$ & innate immune response \\
\hline GO:0042110 & $8.62 \mathrm{e}-20$ & $1.21 \mathrm{e}-17$ & T cell activation \\
\hline \multicolumn{4}{|c|}{ HULC (liver-related processes) } \\
\hline GO:0002933 & $0.00 \mathrm{e}+00$ & $0.00 \mathrm{e}+00$ & lipid hydroxylation \\
\hline GO:0006547 & $0.00 \mathrm{e}+00$ & $0.00 \mathrm{e}+00$ & histidine metabolic process \\
\hline GO:0006572 & $0.00 \mathrm{e}+00$ & $0.00 \mathrm{e}+00$ & tyrosine catabolic process \\
\hline GO:0010873 & $0.00 \mathrm{e}+00$ & $0.00 \mathrm{e}+00$ & positive regulation of cholesterol esterification \\
\hline GO:0010898 & $0.00 \mathrm{e}+00$ & $0.00 \mathrm{e}+00$ & positive regulation of triglyceride catabolic process \\
\hline GO:0016098 & $0.00 \mathrm{e}+00$ & $0.00 \mathrm{e}+00$ & monoterpenoid metabolic process \\
\hline GO:0030300 & $0.00 \mathrm{e}+00$ & $0.00 \mathrm{e}+00$ & regulation of intestinal cholesterol absorption \\
\hline GO:0034371 & $0.00 \mathrm{e}+00$ & $0.00 \mathrm{e}+00$ & chylomicron remodelling \\
\hline GO:0034378 & $0.00 \mathrm{e}+00$ & $0.00 \mathrm{e}+00$ & chylomicron assembly \\
\hline GO:0042737 & $0.00 \mathrm{e}+00$ & $0.00 \mathrm{e}+00$ & drug catabolic process \\
\hline \multicolumn{4}{|c|}{ H19 (cancer-related processes) } \\
\hline GO:0007565 & $3.46 \mathrm{e}-14$ & $2.79 \mathrm{e}-11$ & female pregnancy \\
\hline GO:0060397 & $7.14 \mathrm{e}-08$ & $1.92 \mathrm{e}-05$ & $\begin{array}{l}\text { JAK-STAT cascade involved in growth hormone } \\
\text { signaling pathway }\end{array}$ \\
\hline GO:0070234 & $1.95 \mathrm{e}-06$ & $3.93 e-04$ & positive regulation of $\mathrm{T}$ cell apoptotic process \\
\hline GO:0007292 & $3.90 e-05$ & $3.50 \mathrm{e}-03$ & female gamete generation \\
\hline GO:0016486 & $3.90 e-05$ & $3.50 \mathrm{e}-03$ & peptide hormone processing \\
\hline GO:0030325 & $3.90 e-05$ & $3.50 \mathrm{e}-03$ & adrenal gland development \\
\hline
\end{tabular}


Table 3 Top 10 biological processes assigned to each of the selected case studies (Continued)

\begin{tabular}{clll}
\hline GO ID & $P$-value & FDR & Term \\
\hline GO:0007267 & $5.08 \mathrm{e}-05$ & $4.31 \mathrm{e}-03$ & cell-cell signalling \\
GO:0042060 & $8.28 \mathrm{e}-05$ & $5.50 \mathrm{e}-03$ & wound healing \\
GO:0006703 & $1.55 \mathrm{e}-04$ & $8.91 \mathrm{e}-03$ & estrogen biosynthetic process \\
GO:0030540 & $4.66 \mathrm{e}-04$ & $1.75 \mathrm{e}-02$ & female genitalia development \\
\hline
\end{tabular}

(GO:0000183) and nucleosome assembly (GO:0006334), which may be consistent with observations that LINC-ROR is involved in regulation of differentiation of embryonic stem cells [53].

We also tested the performance on a more general data set, using a set of prostate cancer-associated lncRNAs with unknown molecular mechanism, published by Mitobe et al. [54]. Most of these lncRNAs are upregulated in prostate cancer, and it has been shown that RNA interference (RNAi) towards these lncRANs leads to a reduction in proliferation, making it reasonable to assume that their upregulation in prostate cancer contributes to increased proliferation in cancer tissue. However, function prediction on this gene set illustrates one of the main challenges. The lack of proper benchmark data, and the fact that the molecular mechanism for these lncRNAs is unknown, makes it difficult to assess the quality of the predictions. There are some cases where recent results seem to support some of the predictions. For example, for SNHG1 (Small nucleolar RNA host gene 1) a highly significant prediction is for positive regulation of histone $\mathrm{H} 3-\mathrm{K} 27$ methylation (GO: 0061087), which is consistent with recent results from $\mathrm{Yu}$ et al. [55] showing that SNHG1 may be involved in epigenetic silencing of the tumour suppressor CDKN1A. However, in most cases the predictions need further verification. The prediction output is therefore included here as supplementary material for future assessment (see Additional file 4: Table S3).

\section{Discussion}

In recent years, thousands of lncRNAs have been discovered that probably play important roles in many different biological processes and diseases, but unfortunately the vast majority of them still need to be functionally annotated. In this paper, we present an improved approach for estimating co-expression for computational function prediction. We compare several measures for estimating co-expression, covering both statistical and geometrical ones, and this gives improved identification of true co-expression. We use an enrichment analysis to identify enriched GO terms in the co-expressed gene set, and use this to predict GO terms for un-annotated genes. This can be any un-annotated gene, but here in particular human lncRNAs.
We have benchmarked the co-expression for enrichment analysis on a subset of well-annotated protein-coding genes. For each gene the GO terms were predicted (without using the known terms for the gene), and the fit between predicted and known GO terms was measured using semantic similarity measures. This showed good correlation between predicted and known GO terms, in particular for terms related to biological process (BP) and when using a combined similarity measure on gene expression. These score values are clearly better than the score values achieved using Pearson or Spearman, which previously has been the most common approach. The procedure was then tested on lncRNAs, in particular using a set of five well-described lncRNAs tested in previous publications [22]. The predicted GO-terms showed good correspondence with published functional descriptions of these lncRNAs. This shows that it is possible to predict the function of both protein-coding and ncRNA genes, given a reliable set of expression data.

There is still room for improvement. Although the prediction is successful in many cases (indicated by the high average similarity score in the benchmarking), the high SD indicates that there are specific cases where the prediction is less successful. It would be very useful if we were able to identify and focus on cases where prediction is most likely to be successful. The performance may also be sensitive to the quality, variation and annotation of the reference data. The approach used for enrichment analysis will also influence the result.

\section{Conclusion}

The results presented here show that approaches for computational gene annotation based on co-expressed genes can provide useful annotations, in particular when using improved estimates of co-expression based on a combination of geometrical and statistical metrics.

\section{Additional files}

Additional file 1: Figure S1. A flowchart illustrating the approach used for prediction and benchmarking in LNCRNA2GOA. (PDF $342 \mathrm{~kb}$ )

Additional file 2: Table S1. Predicted annotation for a set of IncRNAs from HeLa, previously analysed with IncFunTK by Zhou et al. [27] and listed in their Table S2. (TXT $76 \mathrm{~kb}$ )

Additional file 3: Table S2. Predicted annotations from Additional file 3: Table S1 for IncRNAs found in PubMed, illustrated with selected PubMed references. (TXT 435 kb) 
Additional file 4: Table S3. Predicted annotation for a set of IncRNAs with unknown mechanism as described by Mitobe et al. [54] in their Table 1 and discussed in the main paper. (PDF 475 kb)

\section{Abbreviations}

(I)ncRNA: (long) non-coding RNA; BP: Biological process; GO: Gene ontology; MF: Molecular function; SD: Standard deviation

\section{Acknowledgements}

Not applicable.

\section{Funding}

This work has been supported by NTNU - Norwegian University of Science and Technology, Department of Clinical and Molecular Medicine (RE), and by University of Zabol (RE). The funding bodies have not been involved in design of the study, in collection, analysis or interpretation of data, or in writing the manuscript.

\section{Availability of data and materials}

A software implementation in $\mathrm{R}$ is available for download via GitHub, at [38], together with suitable data files with expression data and GO terms. The most recent version of TopolCSim is also available for download via GitHub, at [56]

\section{Consent to publish}

Not applicable.

\section{Authors' contributions}

RE developed the methodology, implemented the software, did the benchmarking and drafted the manuscript. FD initiated the project and evaluated test cases. Both authors contributed to and approved the final manuscript.

\section{Ethics approval and consent to participate}

Not applicable.

\section{Competing interests}

The authors declare that they have no competing interests.

\section{Publisher's Note}

Springer Nature remains neutral with regard to jurisdictional claims in published maps and institutional affiliations.

\section{Author details}

'Department of Mathematics, University of Zabol, Zabol, Iran. ${ }^{2}$ Department of Bioinformatics, University of Zabol, Zabol, Iran. ${ }^{3}$ Department of Clinical and Molecular Medicine, NTNU - Norwegian University of Science and Technology, NO-7491 Trondheim, Norway.

\section{Received: 7 August 2018 Accepted: 28 November 2018}

Published online: 19 December 2018

\section{References}

1. Kung JT, Colognori D, Lee JT. Long noncoding RNAs: past, present, and future. Genetics. 2013;193(3):651-69.

2. Dimitrova N, Zamudio JR, Jong RM, Soukup D, Resnick R, Sarma K, Ward AJ, Raj A, Lee JT, Sharp PA, et al. LincRNA-p21 activates p21 in cis to promote Polycomb target gene expression and to enforce the G1/S checkpoint. Mol Cell. 2014;54(5):777-90.

3. Guttman M, Donaghey J, Carey BW, Garber M, Grenier JK, Munson G, Young $G$, Lucas $A B, A$ ch $R$, Bruhn $L$, et al. lincRNAs act in the circuitry controlling pluripotency and differentiation. Nature. 2011;477(7364):295-300.

4. Barnhill LM, Williams RT, Cohen O, Kim Y, Batova A, Mielke JA, Messer K, Pu M, Bao L, Yu AL, et al. High expression of CAl2, a 9p21-embedded long noncoding RNA, contributes to advanced-stage neuroblastoma. Cancer Res. 2014;74(14):3753-63.

5. Chen G, Wang Z, Wang D, Qiu C, Liu M, Chen X, Zhang Q, Yan G, Cui Q. LncRNADisease: a database for long-non-coding RNA-associated diseases. Nucleic Acids Res. 2013;41(Database issue):D983-6.
6. Fang Y, Fullwood MJ. Roles, functions, and mechanisms of long non-coding RNAs in Cancer. Genomics Proteomics Bioinformatics. 2016;14(1):42-54

7. Carpenter S, Aiello D, Atianand MK, Ricci EP, Gandhi P, Hall LL, Byron M, Monks B, Henry-Bezy M, Lawrence JB, et al. A long noncoding RNA mediates both activation and repression of immune response genes. Science. 2013;341(6147):789-92.

8. Sun L, Goff LA, Trapnell C, Alexander R, Lo KA, Hacisuleyman E, Sauvageau M, Tazon-Vega B, Kelley DR, Hendrickson DG, et al. Long noncoding RNAs regulate adipogenesis. Proc Natl Acad Sci U S A. 2013;110(9):3387-92.

9. Signal B, Gloss BS, Dinger ME. Computational approaches for functional prediction and characterisation of long noncoding RNAs. Trends Genet. 2016;32(10):620-37.

10. Xiao Y, LV Y, Zhao H, Gong Y, Hu J, Li F, Xu J, Bai J, Yu F, Li X. Predicting the functions of long noncoding RNAs using RNA-seq based on Bayesian network. Biomed Res Int. 2015:2015:839590.

11. Yang $Y$, Wen $L$, Zhu $H$. Unveiling the hidden function of long non-coding RNA by identifying its major partner-protein. Cell Biosci. 2015:5:59.

12. Mercer TR, Dinger ME, Mattick JS. Long non-coding RNAs: insights into functions. Nat Rev Genet. 2009;10(3):155-9.

13. Chu C, Qu K, Zhong FL, Artandi SE, Chang HY. Genomic maps of long noncoding RNA occupancy reveal principles of RNA-chromatin interactions. Mol Cell. 2011;44(4):667-78.

14. Simon MD, Wang $\mathrm{Cl}$, Kharchenko PV, West JA, Chapman BA, Alekseyenko AA, Borowsky ML, Kuroda MI, Kingston RE. The genomic binding sites of a noncoding RNA. Proc Natl Acad Sci U S A. 2011;108(51):20497-502.

15. Eisen MB, Spellman PT, Brown PO, Botstein D. Cluster analysis and display of genome-wide expression patterns. Proc Natl Acad Sci U S A. 1998;95(25): 14863-8.

16. Lee HK, Hsu AK, Sajdak J, Qin J, Pavlidis P. Coexpression analysis of human genes across many microarray data sets. Genome Res. 2004;14(6):1085-94.

17. Alam T, Uludag M, Essack M, Salhi A, Ashoor H, Hanks JB, Kapfer C, Mineta K, Gojobori T, Bajic VB. FARNA: knowledgebase of inferred functions of noncoding RNA transcripts. Nucleic Acids Res. 2017;45(5):2838-48.

18. Gong Y, Huang HT, Liang Y, Trimarchi T, Aifantis I, Tsirigos A. IncRNA-screen: an interactive platform for computationally screening long non-coding RNAs in large genomics datasets. BMC Genomics. 2017:18(1):434.

19. Guttman M, Amit I, Garber M, French C, Lin MF, Feldser D, Huarte M, Zuk O, Carey BW, Cassady JP, et al. Chromatin signature reveals over a thousand highly conserved large non-coding RNAs in mammals. Nature. 2009; 458(7235):223-7.

20. Guo X, Gao L, Liao Q, Xiao H, Ma X, Yang X, Luo H, Zhao G, Bu D, Jiao F, et al. Long non-coding RNAs function annotation: a global prediction method based on bi-colored networks. Nucleic Acids Res. 2013;41(2):e35.

21. Liao Q, Liu C, Yuan X, Kang S, Miao R, Xiao H, Zhao G, Luo H, Bu D, Zhao H, et al. Large-scale prediction of long non-coding RNA functions in a coding-noncoding gene co-expression network. Nucleic Acids Res. 2011;39(9):3864-78.

22. Jiang $Q$, Ma R, Wang J, Wu X, Jin S, Peng J, Tan R, Zhang T, Li Y, Wang $Y$. LncRNA2Function: a comprehensive resource for functional investigation of human IncRNAs based on RNA-seq data. BMC Genomics. 2015;16(Suppl 3):S2.

23. Park C, Yu N, Choi I, Kim W, Lee S. IncRNAtor: a comprehensive resource for functional investigation of long non-coding RNAs. Bioinformatics. 2014; 30(17):2480-5.

24. Zhao Z, Bai J, Wu A, Wang Y, Zhang J, Wang Z, Li Y, Xu J, Li X. Co-LncRNA: investigating the IncRNA combinatorial effects in $\mathrm{GO}$ annotations and KEGG pathways based on human RNA-Seq data. Database (Oxford). 2015;2015:bav082.

25. Perron U, Provero $P$, Molineris I. In silico prediction of IncRNA function using tissue specific and evolutionary conserved expression. BMC Bioinformatics. 2017;18(Suppl 5):144.

26. Zhou J, Zhang S, Wang H, Sun H. LncFunNet: an integrated computational framework for identification of functional long noncoding RNAs in mouse skeletal muscle cells. Nucleic Acids Res. 2017:45(12):e108.

27. Zhou J, Huang Y, Ding Y, Yuan J, Wang H, Sun H. IncFunTK: a toolkit for functional annotation of long noncoding RNAs. Bioinformatics. 2018;34(19): 3415-6.

28. Zhang J, Zhang Z, Wang Z, Liu Y, Deng L. Ontological function annotation of long non-coding RNAs through hierarchical multi-label classification. Bioinformatics. 2018:34(10):1750-7.

29. Harrow J, Frankish A, Gonzalez JM, Tapanari E, Diekhans M, Kokocinski F, Aken BL, Barrell D, Zadissa A, Searle S, et al. GENCODE: the reference human genome annotation for the ENCODE project. Genome Res. 2012;22(9):1760-74. 
30. Kim D, Pertea G, Trapnell C, Pimentel H, Kelley R, Salzberg SL. TopHat2: accurate alignment of transcriptomes in the presence of insertions, deletions and gene fusions. Genome Biol. 2013;14(4):R36.

31. Trapnell C, Williams BA, Pertea G, Mortazavi A, Kwan G, van Baren MJ, Salzberg SL, Wold BJ, Pachter L. Transcript assembly and quantification by RNA-Seq reveals unannotated transcripts and isoform switching during cell differentiation. Nat Biotechnol. 2010;28(5):511-5.

32. Ashburner M, Ball CA, Blake JA, Botstein D, Butler H, Cherry JM, Davis AP, Dolinski K, Dwight SS, Eppig JT, et al. Gene ontology: tool for the unification of biology. Nat Genet. 2000;25(1):25-9.

33. R Core Team. R: a language and environment for statistical computing. Vienna, Austria: R Foundation for statistical Computing; 2018.

34. Wickham $\mathrm{H}$. The Split-apply-combine strategy for data analysis. J Stat Softw. 2011;40(1):1-29.

35. Frohlich H, Speer N, Poustka A, Beissbarth T. GOSim--an R-package for computation of information theoretic $\mathrm{GO}$ similarities between terms and gene products. BMC Bioinformatics. 2007;8:166.

36. Villmann T. Sobolev metrics for learning of functional data - mathematical and theoretical aspects. In: Villmann T, Schleif F-M, editors. Machine Learning Reports, vol. vol. 1. Leipzig, Germany: Medical Department, University of Leipzig; 2007. p. 1-13.

37. Lebanon G. Learning riemannian metrics. In: Proceedings of the Nineteenth conference on Uncertainty in Artificial Intelligence. Acapulco, Mexico: Morgan Kaufmann Publishers Inc; 2003. p. 362-9.

38. LNCRNA2GOA [https://github.com/RezvanEhsani/LNCRNA2GOA].

39. Ehsani R, Drablos F. TopolCSim: a new semantic similarity measure based on gene ontology. BMC Bioinformatics. 2016;17(1):296.

40. Yu G, Li F, Qin Y, Bo X, Wu Y, Wang S. GOSemSim: an R package for measuring semantic similarity among $\mathrm{GO}$ terms and gene products. Bioinformatics. 2010;26(7):976-8.

41. Bhan A, Mandal SS. LncRNA HOTAIR: a master regulator of chromatin dynamics and cancer. Biochim Biophys Acta. 2015;1856(1):151-64.

42. Woo CJ, Kingston RE. HOTAIR lifts noncoding RNAs to new levels. Cell. 2007; 129(7):1257-9.

43. van Manen D, Kootstra NA, Boeser-Nunnink B, Handulle MA, van't Wout AB, Schuitemaker $\mathrm{H}$. Association of HLA-C and HCP5 gene regions with the clinical course of HIV-1 infection. AIDS. 2009;23(1):19-28.

44. Lange CM, Bibert S, Dufour JF, Cellerai C, Cerny A, Heim MH, Kaiser L, Malinverni R, Mullhaupt B, Negro F, et al. Comparative genetic analyses point to HCP5 as susceptibility locus for HCV-associated hepatocellular carcinoma. J Hepatol. 2013;59(3):504-9.

45. Ma Z, Huang H, Xu Y, He X, Wang J, Hui B, Ji H, Zhou J, Wang K. Current advances of long non-coding RNA highly upregulated in liver cancer in human tumors. Onco Targets Ther. 2017;10:4711-7.

46. Panzitt K, Tschernatsch MM, Guelly C, Moustafa T, Stradner M, Strohmaier HM, Buck CR, Denk H, Schroeder R, Trauner M, et al. Characterization of HULC, a novel gene with striking up-regulation in hepatocellular carcinoma, as noncoding RNA. Gastroenterology. 2007;132(1):330-42.

47. Gabory A, Jammes H, Dandolo L. The H19 locus: role of an imprinted noncoding RNA in growth and development. Bioessays. 2010;32(6):473-80.

48. Gabory A, Ripoche MA, Yoshimizu T, Dandolo L. The H19 gene: regulation and function of a non-coding RNA. Cytogenet Genome Res. 2006;113(1-4): 188-93.

49. Ferreira LB, Palumbo A, de Mello KD, Sternberg C, Caetano MS, de Oliveira FL, Neves AF, Nasciutti LE, Goulart LR, Gimba ER. PCA3 noncoding RNA is involved in the control of prostate-cancer cell survival and modulates androgen receptor signaling. BMC Cancer. 2012;12:507.

50. Lemos AE, Ferreira LB, Batoreu NM, de Freitas PP, Bonamino MH, Gimba ER. PCA3 long noncoding RNA modulates the expression of key cancer-related genes in LNCaP prostate cancer cells. Tumour Biol. 2016;37(8):11339-48.

51. Tang PM, Zhang YY, Mak TS, Tang PC, Huang XR, Lan HY. Transforming growth factor-beta signalling in renal fibrosis: from Smads to non-coding RNAs. J Physiol. 2018;596(16):3493-503.

52. Hu XL, Wang J, He W, Zhao P, Wu WQ. Down-regulation of IncRNA Linc00152 suppressed cell viability, invasion, migration, and epithelial to mesenchymal transition, and reversed chemo-resistance in breast cancer cells. Eur Rev Med Pharmacol Sci. 2018;22(10):3074-84.

53. Wang Y, Xu Z, Jiang J, Xu C, Kang J, Xiao L, Wu M, Xiong J, Guo X, Liu H. Endogenous miRNA sponge lincRNA-RoR regulates Oct4, Nanog, and Sox2 in human embryonic stem cell self-renewal. Dev Cell. 2013;25(1):69-80.
54. Mitobe Y, Takayama Kl, Horie-Inoue K, Inoue S. Prostate cancer-associated IncRNAs. Cancer Lett. 2018;418:159-66.

55. Yu Y, Zhang M, Wang N, Li Q, Yang J, Yan S, He X, Ji G, Miao L. Epigenetic silencing of tumor suppressor gene CDKN1A by oncogenic long noncoding RNA SNHG1 in cholangiocarcinoma. Cell Death Dis. 2018;9(7):746.

56. TopolCSim [https://github.com/RezvanEhsani/TopolCSim].
Ready to submit your research? Choose BMC and benefit from:

- fast, convenient online submission

- thorough peer review by experienced researchers in your field

- rapid publication on acceptance

- support for research data, including large and complex data types

- gold Open Access which fosters wider collaboration and increased citations

- maximum visibility for your research: over $100 \mathrm{M}$ website views per year

At BMC, research is always in progress.

Learn more biomedcentral.com/submissions 